\title{
CARACTERIZAÇÃO E CLASSIFICAÇÃO DO RESÍDUO SÓLIDO “PÓ DO BALÃO”, GERADO NA INDÚSTRIA SIDERÚRGICA NÃO INTEGRADA A CARVÃO VEGETAL:- ESTUDO DE UM CASO NA REGIÃO DE SETE LAGOAS/MG
}

\section{Míriam Regina Cardoso de Oliveira*}

Fundação Estadual do Meio Ambiente, Av. Prudente de Morais, 1671, 30380-000 Belo Horizonte - MG

Jader Martins

Departamento de Engenharia de Minas, Escola de Minas, Universidade Federal de Ouro Preto, Morro do Cruzeiro, 35400-000 Ouro Preto - MG

Recebido em 11/6/01; aceito em 8/7/02

\begin{abstract}
CARACTERIZATION AND CLASSIFICATION OF SOLID WASTE "PÓ DO BALÃO", PRODUCED BY MERCHANT PIG IRON INDUSTRY WHICH USE CHARCOAL AS ENERGY SOURCE IN BRASIL: CASE STUDY OF SETE LAGOAS REGION, STATE OF MINAS GERAIS. This research was developed by considering that the solid waste produced in the process of pig iron production represents the loss of raw materials and the increase in environmental problem. The charcoal based mini blastfurnace off gases dust named CHARCOK was collected from SIDERPA - Siderúrgica Paulino Ltda, located in Sete Lagoas, Minas Gerais. The Charcok was characterized and classified according to ABNT (Associação Brasileira de Normas Técnicas) standard. The results showed that the Charcok should be classified as Class I Wastes - "Hazard Wastes" because of its high concentration of phenols $\left(54.5 \mathrm{mg} \mathrm{C}_{6} \mathrm{H}_{5} \mathrm{OH} / \mathrm{kg}\right)$. The Charcok had high concentration of iron and charcoal which can be used as energy source.
\end{abstract}

Keywords: solid waste; pig iron; phenols.

\section{INTRODUÇÃO}

Os resíduos sólidos - industriais e urbanos - merecem cada vez mais atenção de especialistas e do poder público dos países que se dedicam ao trabalho de melhoria da qualidade ambiental. Todos os países, não importando sua localização ou seu "status" internacional, produzem milhões de toneladas por dia de resíduos, o que justifica a obrigatoriedade da criação de mecanismos que produzam a conscientização, o desenvolvimento e a implantação de tecnologias para reverter este quadro.

A aplicação de tecnologias apropriadas e ecologicamente corretas, provocando assim uma redução da utilização de recursos naturais, de desperdício, da geração de resíduos e poluição, é uma ação de prioridade mundial. A produção eficaz e a poluição zero advinda desta é o desafio inerente às estratégias de produção mais limpa, cujo objetivo principal é evitar a geração de resíduos e emissões, a partir de um enfoque preventivo.

Existe uma preocupação em otimizar o uso da biomassa, para gerar menor quantidade de resíduos e garantir sua reciclagem industrial, reintegrando materiais residuais ao ciclo produtivo de forma a evitar gastos com tratamento e disposição; incentivando a promoção de tecnologias limpas e o uso racional de matéria-prima natural, é o grande desafio mundial para este novo milênio.

Especificamente, esta pesquisa trata do resíduo sólido conhecido como pó do balão coletor do alto-forno - pó do sistema de limpeza à seco dos gases do alto-forno - (Figura 1), que foi denominado de "Charcok" (os autores introduziram este nome para o resíduo pó do balão, oriundo da indústria siderúrgica não integrada a carvão vegetal, que utiliza o carvão vegetal (Charcoal) como redutor; existem outros resíduos à base de Carbono que tem nomes similares

*e-mail: miriamr@feam.br

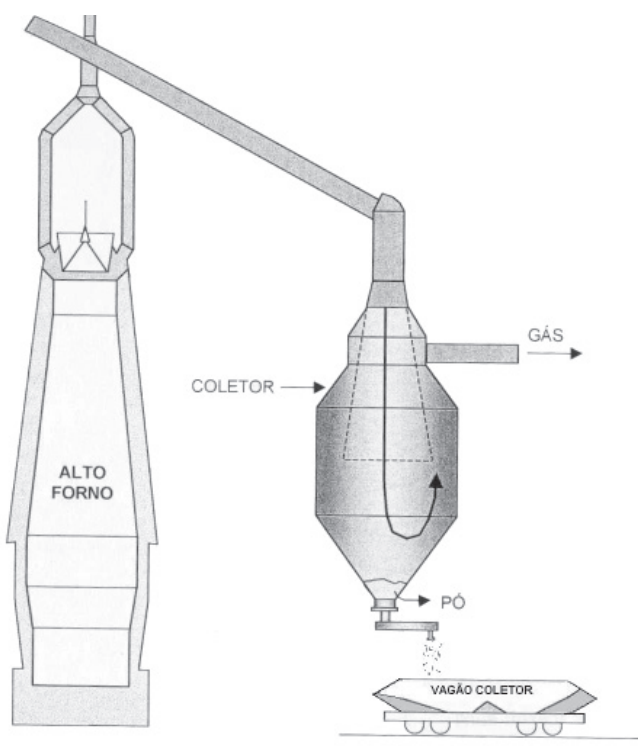

Figura 1. Esquema do coletor de pó de balão para limpeza do gás de topo do alto-forno

(Fercoque e Alucoque) e corresponde a 13\% dos resíduos sólidos gerados nas indústrias siderúrgicas independentes.

\section{PARTE EXPERIMENTAL}

\section{Antecedentes}

As siderúrgicas mineiras enfrentam problemas com as emissões atmosféricas, com os efluentes líquidos e, principalmente, com os 
resíduos sólidos. Sabe-se que a destinação de 74\% do resíduo sólido 1 "pó do balão do alto-forno/pó do balão", se dá a céu aberto nos pátios das empresas, possibilitando a contaminação do solo e dos corpos d'água locais e ainda, que a falta de políticas e diretrizes para o gerenciamento de resíduos sólidos industriais constitui um dos problemas ambientais mais graves, com o qual o setor siderúrgico tem se deparado nos últimos tempos.

Segundo o projeto Minas Ambiente ${ }^{2}$, o pó do alto-forno (resíduo Charcok) consiste no material particulado carreado do forno pelos gases efluentes e que é retido em um sistema de limpeza à seco de gases. A quantidade vai depender do processo e da eficiência do sistema de controle. Os produtores independentes no estado de $\mathrm{Mi}$ nas Gerais, geram de 28 a $45 \mathrm{~kg}$ de Charcok/t gusa e a região de Sete Lagoas é uma das principais do estado.

A composição do material particulado varia com o processo produtivo e com as matérias-primas empregadas. A composição química aproximada do Resíduo Charcok é apresentada na Tabela 1.

Tabela 1. Composição Química aproximada do Resíduo Charcok

\begin{tabular}{cc}
\hline ELEMENTOS & PERCENTAGEM (\%) \\
\hline $\mathrm{Fe}_{2} \mathrm{O}_{3}$ & 57 \\
$\mathrm{SiO}_{2}$ & 12 \\
$\mathrm{Al}_{2} \mathrm{O}_{3}$ & 2,0 \\
$\mathrm{P}_{2} \mathrm{O}_{5}$ & 0,05 \\
$\mathrm{CaO}$ & 3,0 \\
$\mathrm{MgO}$ & 0,1 \\
Carvão Vegetal & 20 \\
\hline
\end{tabular}

\section{Materiais e Métodos}

O resíduo Charcok foi coletado diretamente da caçamba receptora e após ser coletado, a amostra foi armazenada em "big-bags", tendose a preocupação de mantê-lo em ambiente livre de sol e chuva, visando preservar suas características iniciais. Algumas vezes por questões técnicas, o processo produtivo foi interrompido e por esta razão não foi possível coletar as 4 amostras diárias, o que resultou num total de 82 amostras.

Após a homogeneização e o quarteamento das amostras, foi retirada a amostra composta, perfazendo um total de 10 frascos, com aproximadamente $2 \mathrm{~kg}$ em cada frasco.

As amostras de resíduos Charcok (semi-compostas) foram enviadas ao laboratório de análises químicas da Siderpa, para análise de alguns parâmetros e a amostra composta foi encaminhada para as devidas análises químicas e físicas necessárias para se proceder à caracterização e classificação do resíduo Charcok. O procedimento de amostragem seguiu a norma da ABNT - NBR $10007^{3}$.

A Tabela 2 apresenta os resultados da análise de fluorescência de raios-X para o resíduo Charcok.

Os resultados da análise do resíduo Charcok são apresentados na Tabela 3 e foram obtidos a partir dos ensaios de queima do resí-

Tabela 2. Caracterização do resíduo Charcok por fluorescência X

\begin{tabular}{lccc}
\hline \multicolumn{3}{c}{ Caracterização por fluorescência de raios - X } \\
\hline \multirow{2}{*}{$\begin{array}{l}\text { Identificação } \\
\text { da amostra }\end{array}$} & $\begin{array}{c}\text { Análise Química Semi-quantitativa } \\
\text { maiores }\end{array}$ & $\begin{array}{c}\text { Elementos } \\
\text { menores }\end{array}$ & $\begin{array}{c}\text { Elementos } \\
\text { traços }\end{array}$ \\
\cline { 2 - 4 } resíduo Charcok & $\mathrm{O}, \mathrm{Fe}$ & $\mathrm{Ca}, \mathrm{Si}$ & $\begin{array}{l}\mathrm{P}, \mathrm{Ti}, \mathrm{K}, \mathrm{Ni}, \mathrm{Zn}, \\
\mathrm{S}, \mathrm{Al}, \mathrm{Mg}, \mathrm{Cl}, \mathrm{Na}\end{array}$ \\
\hline
\end{tabular}

Tabela 3. Análises do resíduo Charcok

\begin{tabular}{lc}
\hline \multicolumn{2}{c}{ Amostra composta de Charcok } \\
\hline Parâmetros analisados & Amostra resíduo Charcok \\
\hline Cinzas (\%) & 70,29 \\
Mat. combustível (MV+CF) (\%) & 29,70 \\
Carbono fixo (\%) & 21,25 \\
Mat. volátil (\%) & 8,45 \\
Carvão vegetal no resíduo (\%) & 30,94 \\
Umidade $(\%)$ & 0,81 \\
PCS $(\mathrm{kJ} / \mathrm{kg})(1 \mathrm{~kJ} / \mathrm{kg}=4,1868 \mathrm{kcal} / \mathrm{kg})$ & 18.581 \\
\hline
\end{tabular}

duo Charcok à $580{ }^{\circ} \mathrm{C}$ para obtenção do teor de cinzas e material combustível, o qual corresponde às frações de Carbono Fixo (CF) e Material Volátil (MV).

A percentagem de carvão vegetal encontrada no resíduo foi estimada considerando-se que o teor de cinzas do carvão vegetal corresponde à $4 \%$ e o teor de $\mathrm{CF}+\mathrm{MV}$ à $96 \%$.

\section{Metodologia para o resíduo Charcok}

Os métodos utilizados nas análises químicas seguiram as recomendações do "Standard Methods for the Examination of Water and Wastewater" " da APHA/AWWA/WEF - $18^{\text {a }}$ edição, 1992 e um conjunto de Normas da ABNT (NBR 1004 ; NBR 1005 ${ }^{6}$; NBR $10006^{7}$ ).

\section{Roteiro de estudo}

Seguindo os critérios descritos nas normas citadas anteriormente, foi estabelecido o seguinte roteiro de estudo:

\section{Pesquisa de periculosidade}

Identificação da origem do resíduo e consulta às Listagens 1 e 2 da NBR 10004. Se o resíduo constar destas listagens, deve ser considerado como PERIGOSO. Outras características que conferem periculosidade a um resíduo são inflamabilidade, corrosividade, reatividade e patogenicidade que não foram estudadas em face da origem conhecida do resíduo em questão e sua forma de apresentação.

Desta forma, para caracterizar a periculosidade ou não do resíduo em questão, foi realizado o teste de lixiviação (Tabela 4), com análise dos parâmetros considerados significativos. A comparação com os limites máximos segundo os parâmetros pesquisados no Extrato Lixiviado é apresentada na Tabela 5.

Tabela 4. Dados relativos ao ensaio de lixiviação

\begin{tabular}{cc}
\hline \multicolumn{2}{c}{ Teste de lixiviação } \\
\hline $\mathrm{pH}$ inicial & 8,25 \\
$\mathrm{pH}$ final & 5,09 \\
Volume gasto de ácido & 80,0 \\
acético 0,5N (ml) & \\
Tempo $(\mathrm{h})$ & 24 \\
\hline
\end{tabular}

\section{Pesquisa de solubilidade}

Se após o procedimento acima descrito o resíduo não for considerado como perigoso, deverá ser realizado um teste de solubilização para sua classificação como Classe II - Não Inerte ou Classe III Inerte.

Os resíduos enquadrados como Classe II: Não inerte ou Classe 
III: Inerte são assim classificados quando pelo menos um dos parâmetros estiver acima dos limites máximos listados segundo a norma ABNT NBR 10004.

Os parâmetros pesquisados no extrato solubilizado são apresentados na Tabela 6.

Amostra bruta

Os parâmetros pesquisados na amostra bruta conforme a NBR 10004 são apresentados na Tabela 7.

\section{RESULTADOS E DISCUSSÃO}

\section{Avaliação dos resultados segundo as normas da ABNT para} resíduos sólidos

O resíduo não consta das listagens 1 e 2 da NBR 10004, assim sendo, procedeu-se conforme roteiro de estudo descrito anteriormente.

Na Tabela 4 são apresentados os resultados relativos ao ensaio de lixiviação e nas Tabelas 5 e 6 , respectivamente, são apresentados os

Tabela 5. Concentração de elementos químicos no extrato do teste de lixiviação da amostra de resíduo Charcok

\begin{tabular}{|c|c|c|c|c|}
\hline \multirow{2}{*}{$\begin{array}{l}\text { Parâmetros } \\
\text { analisados }\end{array}$} & $\begin{array}{l}\text { RESULTADOS } \\
\text { Concentração no }\end{array}$ & \multicolumn{2}{|c|}{ Limite máximo no lixiviado (mg/L) } & \multirow{2}{*}{$\begin{array}{c}\text { L.D. do método } \\
\text { (Limite Detecção) } \\
(\mathrm{mg} / \mathrm{L})\end{array}$} \\
\hline & $\begin{array}{l}\text { Amostra } \\
\text { resíduo Charcok }\end{array}$ & $\begin{array}{c}\text { NBR } 10004 \\
\text { Anexo G Listagem no } 7\end{array}$ & $\begin{array}{c}\text { PN-1:603.06.008 } \\
\text { Anexo G Listagem no } 7\end{array}$ & \\
\hline Arsênio & $<0,010$ & 5,0 & 5,0 & 0,010 \\
\hline Bário & $<0,30$ & 100 & 100 & 0,30 \\
\hline Cádmio & $<0,004$ & 0,5 & 1,0 & 0,004 \\
\hline Chumbo total & $<0,05$ & 5,0 & 5,0 & 0,05 \\
\hline Crômio total & $<0,016$ & 5,0 & 5,0 & 0,016 \\
\hline Mercúrio & $<0,001$ & 0,1 & 0,2 & 0,001 \\
\hline Prata & $<0,009$ & 5,0 & 5,0 & 0,009 \\
\hline Selênio & $<0,003$ & 1,0 & 1,0 & 0,003 \\
\hline Fluoreto & 0,70 & 150 & 150 & 0,10 \\
\hline
\end{tabular}

Tabela 6. Concentração de elementos químicos no extrato do teste de solubilização da amostra de resíduo Charcok

\begin{tabular}{|c|c|c|c|c|}
\hline \multirow{2}{*}{$\begin{array}{l}\text { Parâmetros } \\
\text { analisados }\end{array}$} & \multirow{2}{*}{$\begin{array}{c}\text { RESULTADOS } \\
\text { Concentração no } \\
\text { solubilizado (mg/L) }\end{array}$} & \multicolumn{2}{|c|}{ Limite máximo no solubilizado (mg/L) } & \multirow{2}{*}{$\begin{array}{c}\text { L.D. do método } \\
\text { (Limite Detecção) } \\
(\mathrm{mg} / \mathrm{L})\end{array}$} \\
\hline & & $\begin{array}{c}\text { NBR } 10004 \\
\text { Anexo H Listagem no } 8\end{array}$ & $\begin{array}{c}\text { PN-1:603.06.008 } \\
\text { Anexo H Listagem no } 8\end{array}$ & \\
\hline Alumínio & $<0,20$ & 0,2 & 0,2 & 0,2 \\
\hline Arsênio & $<0,010$ & 0,05 & 0,05 & 0,010 \\
\hline Bário & $<0,30$ & 1,0 & 1,0 & 0,30 \\
\hline Cádmio & $<0,004$ & 0,005 & 0,005 & 0,004 \\
\hline Cianeto & $<0,017$ & 0,05 & 0,01 & 0,017 \\
\hline Chumbo total & $<0,05$ & 0,05 & 0,05 & 0,05 \\
\hline Cloreto & 51 & 250,0 & 250,0 & 0,5 \\
\hline Cobre & $<0,010$ & 1,0 & 1,0 & 0,010 \\
\hline Crômio total & $<0,016$ & 0,05 & 0,05 & 0,009 \\
\hline Dureza $\left(\mathrm{mg} \mathrm{CaCO}_{3} / \mathrm{L}\right)$ & 90 & 500 & 500 & 1,0 \\
\hline Fenóis & 15,3 & 0,001 & 0,001 & 0,001 \\
\hline Ferro & 0,164 & 0,3 & 0,3 & 0,012 \\
\hline Fluoreto & $<0,10$ & 1,5 & 1,5 & 0,10 \\
\hline Manganês & 0,389 & 0,1 & 0,1 & 0,012 \\
\hline Mercúrio & $<0,001$ & 0,001 & 0,001 & 0,001 \\
\hline Nitrato (mg N/L) & $<0,017$ & 10,0 & 10,0 & 0,017 \\
\hline Prata & $<0,009$ & 0,05 & 0,05 & 0,009 \\
\hline Selênio & $<0,003$ & 0,01 & 0,01 & 0,003 \\
\hline Sódio & 2,34 & 200 & 200,0 & 0,030 \\
\hline Sulfato $\left(\mathrm{mg} \mathrm{SO}_{4} / \mathrm{L}\right)$ & 26 & 400,0 & 400,0 & 2 \\
\hline Surfactantes (tensoativos) & $<0,2$ & 0,2 & 0,2 & 0,20 \\
\hline Zinco & $<0,001$ & 5,0 & 5,0 & 0,001 \\
\hline
\end{tabular}


Tabela 7. Análises físico-químicas na amostra bruta do resíduo Charcok

\begin{tabular}{|c|c|c|c|c|c|}
\hline \multirow[b]{2}{*}{$\begin{array}{l}\text { Parâmetros } \\
\text { analisados }\end{array}$} & \multirow[b]{2}{*}{ Unidades } & \multirow[b]{2}{*}{$\begin{array}{l}\text { Resultados na } \\
\text { amostra bruta }\end{array}$} & \multicolumn{2}{|c|}{ Limite máximo amostra bruta em $(\mathrm{mg} / \mathrm{kg})$} & \multirow{2}{*}{$\begin{array}{c}\text { L.D. do método } \\
\text { (Limite Detecção) } \\
\text { (mg/kg) }\end{array}$} \\
\hline & & & $\begin{array}{c}\text { NBR } 10004 \\
\text { Anexo I Listagem n }{ }^{\circ} 9\end{array}$ & PN 1:603.06.008 & \\
\hline Aluminio & $\mathrm{mg} \mathrm{Al} / \mathrm{kg}$ & 5380 & - & - & \\
\hline Antimônio & $\mathrm{mg} \mathrm{Sb} / \mathrm{kg}$ & 113 & - & 1000 & 50 \\
\hline Arsênio & $\mathrm{mg} \mathrm{As} / \mathrm{kg}$ & $<2,0$ & 1000 & 1000 & 2,0 \\
\hline Bário & $\mathrm{mg} \mathrm{Ba} / \mathrm{kg}$ & $<15$ & - & 20000 & 15 \\
\hline Cádmio & $\mathrm{mg} \mathrm{Cd} / \mathrm{kg}$ & $<0,5$ & - & 200 & 0,5 \\
\hline Cálcio & $\mathrm{mg} \mathrm{Ca} / \mathrm{kg}$ & 24800 & - & - & \\
\hline Chumbo & $\mathrm{mg} \mathrm{Pb} / \mathrm{kg}$ & 81 & $*$ & 2000 & 10 \\
\hline Cianeto & $\mathrm{mg} \mathrm{CN} / \mathrm{kg}$ & 3,5 & 1000 & - & 1,70 \\
\hline Cobalto & $\mathrm{mg} \mathrm{Co} / \mathrm{kg}$ & $<5$ & - & 16000 & 5 \\
\hline Cobre & $\mathrm{mg} \mathrm{Cu} / \mathrm{kg}$ & 3,3 & - & & 2,5 \\
\hline Crômio VI & $\mathrm{mg} \mathrm{Cr} / \mathrm{kg}$ & $<4,5$ & 100 & 1000 & 4,5 \\
\hline Crômio total & $\mathrm{mg} \mathrm{Cr} / \mathrm{kg}$ & 6,4 & - & 5000 & 3,0 \\
\hline Enxofre & $\%$ & $<0,01$ & - & - & \\
\hline Fenóis & $\mathrm{mg} \mathrm{C}_{6} \mathrm{H}_{5} \mathrm{OH} / \mathrm{kg}$ & 54,5 & 10 & - & 0,10 \\
\hline Ferro total & $\mathrm{mg} \mathrm{Fe} / \mathrm{kg}$ & 415000 & - & - & \\
\hline Fluoreto & $\mathrm{mg} \mathrm{F} / \mathrm{kg}$ & 108 & - & 36000 & 100 \\
\hline Magnésio & $\mathrm{mg} \mathrm{Mg} / \mathrm{kg}$ & 398 & - & - & \\
\hline Manganês & $\mathrm{mg} \mathrm{Mn} / \mathrm{kg}$ & 160 & - & - & \\
\hline Mercúrio & $\mathrm{mg} \mathrm{Hg} / \mathrm{kg}$ & $<1,25$ & 100 & 40 & 1,25 \\
\hline Molibdênio & $\mathrm{mg} \mathrm{Mo} / \mathrm{kg}$ & $<10$ & - & 7000 & 10 \\
\hline Níquel & $\mathrm{mg} \mathrm{Ni} / \mathrm{kg}$ & 11,6 & - & 4000 & 1,5 \\
\hline Nitrogênio total & $\mathrm{mg} \mathrm{N} / \mathrm{kg}$ & 681 & - & - & 3,5 \\
\hline Potássio & $\mathrm{mg} \mathrm{K} / \mathrm{kg}$ & 1740 & - & - & \\
\hline Prata & $\mathrm{mg} \mathrm{Ag} / \mathrm{kg}$ & $<2$ & - & 1000 & 2 \\
\hline Selênio & $\mathrm{mg} \mathrm{Se} / \mathrm{kg}$ & $<3,0$ & 100 & 200 & 3,0 \\
\hline Sódio & $\mathrm{mg} \mathrm{Na} / \mathrm{kg}$ & 93,2 & - & - & \\
\hline Tálio & $\mathrm{mg} \mathrm{Tl} / \mathrm{kg}$ & $<100$ & - & 1400 & 100 \\
\hline Titânio & $\mathrm{mg} \mathrm{Ti} / \mathrm{kg}$ & $<200$ & - & - & \\
\hline Vanádio & $\mathrm{mg} \mathrm{V} / \mathrm{kg}$ & $<50$ & 1000 & 4800 & 50 \\
\hline Zinco & $\mathrm{mg} \mathrm{Zn/kg}$ & 39,9 & - & 10000 & 0,5 \\
\hline Óleos e Graxas & $\%$ & 0,74 & - & - & 0,1 \\
\hline Umidade & $\%$ & 0,81 & - & - & 0,01 \\
\hline $\mathrm{pH}$ & - & 7,23 & - & - & - \\
\hline
\end{tabular}

*Chumbo (compostos orgânicos) 100 mg Pb/kg e Chumbo (compostos inorgânicos) 1000 mg Pb/kg

resultados das análises no extrato obtido no teste de lixiviação e os resultados das análises no extrato obtido no teste de solubilização, que confere ao resíduo sólido Charcok, nesta fase a Classificação de não inerte (os resíduos enquadrados como classe II - não inerte, são assim classificados quando pelo menos um dos parâmetros estiver acima dos limites máximos citados na NBR-10004), a continuidade da classificação do resíduo para a amostra bruta é apresentada na Tabela 7.

Os parâmetros pesquisados na amostra bruta, para limites máximos de poluentes na massa bruta de resíduo (os poluentes listados devem ser dosados no resíduo total e, se pelo menos um poluente estiver acima do limite máximo, o resíduo deve ser disposto em instalações adequadas) são os utilizados pelo Ministério do Meio Ambiente - França para classificação de resíduos. Estes valores podem ser usados como parâmetros indicativos para classificação de um resíduo como perigoso.

\section{Discussão}

O resíduo sólido industrial - Pó do balão "CHARCOK" - objeto deste estudo, apresentou um poder calorífico de $18.581 \mathrm{~kJ} / \mathrm{kg}$, semelhante ao poder calorífico do Resíduo de Alto-Forno (RAF- coque -) que é de $18.841 \mathrm{~kJ} / \mathrm{kg}$, sendo que, segundo o relatório da $\mathrm{CEMIG}^{8}$, sobre o "Uso de Energia na Indústria de Cerâmica Vermelha em MG”, o uso de matérias orgânicas combustíveis na massa cerâmica é uma técnica cerâmica que visa a redução do ciclo térmico e a melhoria de qualidade do produto cerâmico, e o RAF (Fercoque), já é utilizado como um alternativo energético por algumas indústrias de cerâmica vermelha. É uma mistura de finos de coque e óxido de ferro, provenientes do sistema de limpeza dos gases de alto-forno das usinas siderúrgicas que utilizam o coque como redutor. $\mathrm{O}$ fercoque é um rejeito industrial aproveitável, como energético na indústria cerâmica vermelha. $\mathrm{O}$ poder calorífico superior médio do fercoque aproveitado da indústria siderúrgica mineira é de $4500 \mathrm{kcal} / \mathrm{kg}$.

\section{CONCLUSÕES}

O resíduo sólido "CHARCOK" - dentro das condições estudadas - foi caracterizado e classificado como Resíduo Perigoso "Classe I", considerando a NBR para amostra bruta, segundo o Ministério do Meio Ambiente da França, que lhe confere esta classificação decorrente da seguinte situação: 
Presença de fenóis acima do limite máximo permitido para o ensaio da Amostra Bruta, segundo NBR 10004.

Por ter sido classificado como resíduo Classe I (PERIGOSO), há de se ter cuidados especiais na estocagem, manuseio, transporte do resíduo;

Poderá ser usado como fonte energética ou como matéria-prima por algum setor industrial, viabilizando seu uso e lhe conferindo nova e nobre aplicação, tendo como vantagem a não emissão de gases sulfurosos como acontece com o Fercoque.

\section{AGRADECIMENTOS}

Os autores agradecem à FAPEMIG (Fundação de Amparo à Pesquisa do Estado de Minas Gerais) pela bolsa de mestrado concedida à M. R. C. Oliveira (CAM - 90101/97), FEAM, UFOP, CETEC, Siderpa e Ecolabor, pelo apoio ao desenvolvimento desta pesquisa.

\section{REFERÊNCIAS}

1. Herdy, T.; Ferro-gusa pode ter produção mais limpa, J. Gazeta Mercantil, $13 / 01 / 2001$.

2. Projeto Minas Ambiente, Pesquisa Tecnológica para Controle Ambiental em Unidades Independentes de Produção de Ferro-Gusa: Diagnóstico, CDTN/CETEC/FEAM/UFMG, Belo Horizonte, 1998.

3. ABNT - Associação Brasileira de Normas Técnicas; Amostragem de Resíduos, NBR-10007, Rio de Janeiro, 1987.

4. Standard Methods for Examination of Water and Wastewater, American Public Health Association (APHA), Washington, 1992.

5. ABNT - Associação Brasileira de Normas Técnicas; Classificação de Resíduos Sólidos, NBR-10004, Rio de Janeiro, 1987.
6. ABNT - Associação Brasileira de Normas Técnicas; Lixiviação de Resíduos Sólidos, NBR-10005, Rio de Janeiro, 1987.

7. ABNT - Associação Brasileira de Normas Técnicas; Solubilização de Resíduos, NBR-10006, Rio de Janeiro, 1987.

8. CEMIG - Companhia Energética de Minas Gerais; Uso de Energia na Indústria de Cerâmica Vermelha em Minas Gerais-1987/1991, Belo Horizonte, 1993.

9. CEMIG - Companhia Energética de Minas Gerais; Uso de Energia na Indústria de Ferro-Gusa Não Integrada em Minas Gerais, Belo Horizonte, 1998.

11. Cerqueira, L; Rev. Saneamento Ambiental 2000, 65, 26.

12. Cerqueira, L.; Alves, F.; Rev. Saneamento Ambiental, 1998, 59, 18.

13. CETEC - Fundação Centro Tecnológico de Minas Gerais; Programa de Pesquisa e Desenvolvimento em Biomassa e Siderurgia a Carvão Vegetal; Descrição Geral, Belo Horizonte, 1992.

14. Almeida, M. L. B.; Rev. SEMEARH 2000, 2, 8.

15. Bowen, H. J. M; Environmental Chemistry of the Elements, Academic Press: London, 1992

16. Christian, G. D.; ANCHAM, $5^{\text {th }}$ ed., John \& Sons: New York, 1994.

17. Figuerêdo, D. V.; Poluição na Indústria Siderúrgica: Processo, Poluentes e Controle das Emissões, Belo Horizonte, 1985.

18. Manahan, S. E.; Environmental Chemistry, Lewis Publishers: Boca Raton, 1994.

19. Neto, E. O.; Espectrofotometria de Absorção Atômica, Belo Horizonte, 1996.

20. Rocca, A. A. C; Iacovone, A. M. M. B.; Barrotti, A. J.; Casarini, D. C.; Gloeden, E.; Straus, E. L.; Romano, J. A.; Ruiz, L. R.; Silva, L. M.; Saito, L. M.; Pires, M. C.; Leão, M. L. G.; Neto, P. P. C.; Colluci, R.; Cunha, R. C. A.; Resíduos Sólidos Industriais, CETESB: São Paulo, 1993, p. 234.

21. SINDIFER Em Workshop Multilateral: Ferro Gusa e Meio Ambiente; a indústria possível na perspectiva do desenvolvimento sustentável; Belo Horizonte, 1999 